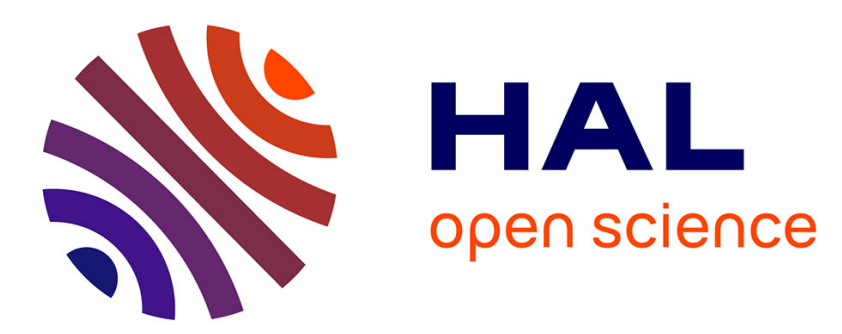

\title{
Distributed lifetime coverage optimization protocol in wireless sensor networks
}

Ali Kadhum Idrees, Karine Deschinkel, Michel Salomon, Raphael Couturier

\section{To cite this version:}

Ali Kadhum Idrees, Karine Deschinkel, Michel Salomon, Raphael Couturier. Distributed lifetime coverage optimization protocol in wireless sensor networks. Journal of Supercomputing, 2015, 71 (12), pp.4578 - 4593. hal-02131127

\section{HAL Id: hal-02131127 \\ https://hal.science/hal-02131127}

Submitted on 16 May 2019

HAL is a multi-disciplinary open access archive for the deposit and dissemination of scientific research documents, whether they are published or not. The documents may come from teaching and research institutions in France or abroad, or from public or private research centers.
L'archive ouverte pluridisciplinaire HAL, est destinée au dépôt et à la diffusion de documents scientifiques de niveau recherche, publiés ou non, émanant des établissements d'enseignement et de recherche français ou étrangers, des laboratoires publics ou privés. 


\title{
Distributed Lifetime Coverage Optimization Protocol in Wireless Sensor Networks
}

\author{
Ali Kadhum Idrees ${ }^{a, b}$, Karine Deschinkel ${ }^{a}$, \\ Michel Salomon ${ }^{a}$, and Raphaël Couturier ${ }^{a}$ \\ ${ }^{a}$ FEMTO-ST Institute, UMR 6174 CNRS, \\ University Bourgogne Franche-Comté, Belfort, France \\ ${ }^{b}$ Department of Computer Science, University of Babylon, Babylon, Iraq \\ email: ali.idness@edu.univ-fcomte.fr, \\ \{karine.deschinkel, michel.salomon, raphael.couturier\}@univ-fcomte.fr
}

October 28,2015

\begin{abstract}
One of the main research challenges faced in Wireless Sensor Networks (WSNs) is to preserve continuously and effectively the coverage of an area (or region) of interest to be monitored, while simultaneously preventing as much as possible a network failure due to battery-depleted nodes. In this paper we propose a protocol, called Distributed Lifetime Coverage Optimization protocol (DiLCO), which maintains the coverage and improves the lifetime of a wireless sensor network. First, we partition the area of interest into subregions using a classical divide-and-conquer method. Our DiLCO protocol is then distributed on the sensor nodes in each subregion in a second step. To fulfill our objective, the proposed protocol combines two effective techniques: a leader election in each subregion, followed by an optimization-based node activity scheduling performed by each elected leader. This two-step process takes place periodically, in order to choose a small set of nodes remaining active for sensing during a time slot. Each set is built to ensure coverage at a low energy cost, allowing to optimize the network lifetime. Simulations are conducted using the discrete event simulator OMNET ++ . We refer to the characterictics of a Medusa II sensor for the energy consumption and the computation time. In comparison with two other existing methods, our approach is able to increase the WSN lifetime and provides improved coverage performances.
\end{abstract}

\section{INTRODUCTION}

Energy efficiency is a crucial issue in wireless sensor networks since sensory consumption, in order to maximize the network lifetime, represents the major difficulty when designing WSNs. As a consequence, one of the scientific research challenges in WSNs, which has been addressed by a large amount of literature during the last few years, is the design of energy efficient approaches for coverage and connectivity [7]. Coverage reflects how well a sensor field is monitored. On the one hand we want to monitor the area of interest in the most efficient way [19], which means that we want to maintain the best coverage as long as possible. On the other hand we want to use as little energy as possible. Sensor nodes are battery-powered with no means of recharging or replacing, usually due to environmental (hostile or unpractical environments) or cost reasons. Therefore, it is desired that the WSNs are deployed with high densities so as to exploit the overlapping sensing regions of some sensor nodes to save energy by turning off some of them during the sensing phase to prolong the network lifetime. A WSN can use various types of sensors such as [1,2]: thermal, seismic, magnetic, visual, infrared, acoustic, and radar. These 
sensors are capable of observing different physical conditions such as: temperature, humidity, pressure, speed, direction, movement, light, soil makeup, noise levels, presence or absence of certain kinds of objects, and mechanical stress levels on attached objects. Consequently, there is a wide range of WSN applications such as [25]: health-care, environment, agriculture, public safety, military, transportation systems, and industry applications.

In this paper we design a protocol that focuses on the area coverage problem with the objective of maximizing the network lifetime. Our proposition, the Distributed Lifetime Coverage Optimization (DiLCO) protocol, maintains the coverage and improves the lifetime in WSNs. The area of interest is first divided into subregions using a divide-and-conquer algorithm and an activity scheduling for sensor nodes is then planned by the elected leader in each subregion. In fact, the nodes in a subregion can be seen as a cluster where each node sends sensing data to the cluster head or the sink node. Furthermore, the activities in a subregion/cluster can continue even if another cluster stops due to too many node failures. Our DiLCO protocol considers periods, where a period starts with a discovery phase to exchange information between sensors of the same subregion, in order to choose in a suitable manner a sensor node (the leader) to carry out the coverage strategy. In each subregion the activation of the sensors for the sensing phase of the current period is obtained by solving an integer program. The resulting activation vector is broadcast by a leader to every node of its subregion.

Our previous paper [11] relies almost exclusively on the framework of the DiLCO approach and the coverage problem formulation. In this paper we made more realistic simulations by taking into account the characteristics of a Medusa II sensor [23] to measure the energy consumption and the computation time. We have implemented two other existing and distributed approaches (DESK [29], and GAF [31]) in order to compare their performances with our approach. We focused on DESK and GAF protocols for two reasons. First our protocol is inspired by both of them: DiLCO uses a regular division of the area of interest as in GAF and a temporal division in rounds as in DESK. Second, DESK and GAF are well-known protocols, easy to implement, and often used as references for comparison. We also focus on performance analysis based on the number of subregions.

The remainder of the paper continues with Section 2 where a review of some related works is presented. The next section describes the DiLCO protocol, followed in Section 4 by the coverage model formulation which is used to schedule the activation of sensors. Section 5 shows the simulation results. The paper ends with a conclusion and some suggestions for further work in Section 6.

\section{LITERATURE REVIEW}

In this section, we summarize some related works regarding the coverage problem and distinguish our DiLCO protocol from the works presented in the literature.

The most discussed coverage problems in literature can be classified into three types [15]: area coverage [18] where every point inside an area is to be monitored, target coverage [32] where the main objective is to cover only a finite number of discrete points called targets, and barrier coverage [14][13] to prevent intruders from entering into the region of interest. In [8] authors transform the area coverage problem to the target coverage problem taking into account the intersection points among disks of sensors nodes or between disk of sensor nodes and boundaries. In DiLCO protocol, the area coverage, i.e. the coverage of every point in the sensing region, is transformed to the coverage of a fraction of points called primary points.

The major approach to extend network lifetime while preserving coverage is to divide/organize the sensors into a suitable number of set covers (disjoint or non-disjoint), where each set completely covers a region of interest, and to activate these set covers successively. The network activity can be planned in advance and scheduled for the entire network lifetime or organized in periods, and the set of active sensor nodes is decided at the beginning of each period [16]. Active node selection is determined based on the problem requirements (e.g. area monitoring, connectivity, power efficiency). For instance, Jaggi et al. [12] address the problem of maximizing network lifetime by dividing sensors into the maximum number of disjoint subsets so that each subset can ensure both coverage and connectivity. A greedy algorithm is applied 
once to solve this problem and the computed sets are activated in succession to achieve the desired network lifetime. $\mathrm{Vu}$ [28], Padmatvathy et al. [20], propose algorithms working in a periodic fashion where a cover set is computed at the beginning of each period. Motivated by these works, DiLCO protocol works in periods, where each period contains a preliminary phase for information exchange and decisions, followed by a sensing phase where one cover set is in charge of the sensing task.

Various approaches, including centralized, or distributed algorithms, have been proposed to extend the network lifetime. In distributed algorithms [33, 29, 22], information is disseminated throughout the network and sensors decide cooperatively by communicating with their neighbors which of them will remain in sleep mode for a certain period of time. The centralized algorithms $[4,36,17]$ always provide nearly or close to optimal solution since the algorithm has global view of the whole network. But such a method has the disadvantage of requiring high communication costs, since the node (located at the base station) making the decision needs information from all the sensor nodes in the area and the amount of information can be huge. In order to be suitable for large-scale network, in the DiLCO protocol, the area coverage is divided into several smaller subregions, and in each one, a node called the leader is in charge for selecting the active sensors for the current period.

Our approach to select the leader node in a subregion is quite different from cluster head selection methods used in LEACH [10] or its variants [24]. Contrary to LEACH, the division of the area of interest is supposed to be performed before the leader election. Moreover, we assume that the sensors are deployed almost uniformly and with high density over the area of interest, so that the division is fixed and regular. As in LEACH, our protocol works in round fashion. In each round, during the pre-sensing phase, nodes make autonomous decisions. In LEACH, each sensor elects itself to be a cluster head, and each non-cluster head will determine its cluster for the round. In our protocol, nodes in the same subregion select their leader. In both protocols, the amount of remaining energy in each node is taken into account to promote the nodes that have the most energy to become leader. Contrary to the LEACH protocol where all sensors will be active during the sensing-phase, our protocol allows to deactivate a subset of sensors through an optimization process which significantly reduces the energy consumption.

A large variety of coverage scheduling algorithms has been developed. Many of the existing algorithms, dealing with the maximization of the number of cover sets, are heuristics. These heuristics involve the construction of a cover set by including in priority the sensor nodes which cover critical targets, that is to say targets that are covered by the smallest number of sensors $[3,36]$. Other approaches are based on mathematical programming formulations $[5,30,17,34]$ and dedicated techniques (solving with a branch-and-bound algorithms available in optimization solver). The problem is formulated as an optimization problem (maximization of the lifetime or number of cover sets) under target coverage and energy constraints. Column generation techniques, well-known and widely practiced techniques for solving linear programs with too many variables, have also been used $[6,26,9]$. In DiLCO protocol, each leader, in each subregion, solves an integer program with a double objective consisting in minimizing the overcoverage and limiting the undercoverage. This program is inspired from the work of [21] where the objective is to maximize the number of cover sets.

\section{DESCRIPTION OF THE DILCO PROTOCOL}

In this section, we introduce the DiLCO protocol which is distributed on each subregion in the area of interest. It is based on two efficient techniques: network leader election and sensor activity scheduling for coverage preservation and energy conservation, applied periodically to efficiently maximize the lifetime in the network.

\subsection{Assumptions and models}

We consider a sensor network composed of static nodes distributed independently and uniformly at random. A high density deployment ensures a high coverage ratio of the interested area at the start. The nodes are supposed to have homogeneous characteristics from a communication and 
a processing point of view, whereas they have heterogeneous energy provisions. Each node has access to its location thanks, either to a hardware component (like a GPS unit), or a location discovery algorithm.

We consider a boolean disk coverage model which is the most widely used sensor coverage model in the literature. Thus, since a sensor has a constant sensing range $R_{s}$, every space points within a disk centered at a sensor with the radius of the sensing range is said to be covered by this sensor. We also assume that the communication range $R_{c} \geq 2 R_{s}$. In fact, Zhang and Hou [35] proved that if the transmission range fulfills the previous hypothesis, a complete coverage of a convex area implies connectivity among the working nodes in the active mode.

For each sensor we also define a set of points called primary points [11] to approximate the area coverage it provides, rather than working with a continuous coverage. Thus, a sensing disk corresponding to a sensor node is covered by its neighboring nodes if all its primary points are covered. Obviously, the approximation of coverage is more or less accurate according to the number of primary points.

\subsection{Main idea}

We start by applying a divide-and-conquer algorithm to partition the area of interest into smaller areas called subregions and then our protocol is executed simultaneously in each subregion. Sensor nodes are assumed to be deployed almost uniformly over the region and the subdivision of the area of interest is regular.

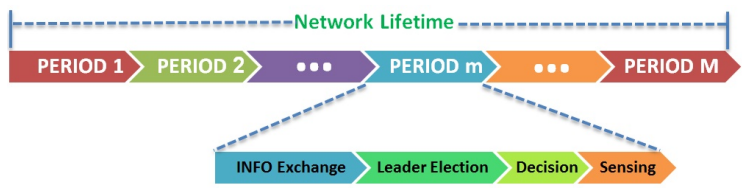

Figure 1: DiLCO protocol

As shown in Figure 1, the proposed DiLCO protocol is a periodic protocol where each period is decomposed into 4 phases: Information Exchange, Leader Election, Decision, and Sensing. For each period there will be exactly one cover set in charge of the sensing task. A periodic scheduling is interesting because it enhances the robustness of the network against node failures. First, a node that has not enough energy to complete a period, or which fails before the decision is taken, will be excluded from the scheduling process. Second, if a node fails later, whereas it was supposed to sense the region of interest, it will only affect the quality of the coverage until the definition of a new cover set in the next period. Constraints, like energy consumption, can be easily taken into consideration since the sensors can update and exchange their information during the first phase. Let us notice that the phases before the sensing one (Information Exchange, Leader Election, and Decision) are energy consuming for all the nodes, even nodes that will not be retained by the leader to keep watch over the corresponding area.

During the execution of the DiLCO protocol, two kinds of packet will be used:

- INFO packet: sent by each sensor node to all the nodes inside a same subregion for information exchange.

- ActiveSleep packet: sent by the leader to all the nodes in its subregion to inform them to stay Active or to go Sleep during the sensing phase.

and each sensor node will have five possible status in the network:

- LISTENING: sensor is waiting for a decision (to be active or not);

- COMPUTATION: sensor applies the optimization process as leader;

- ACTIVE: sensor is active;

- SLEEP: sensor is turned off;

- COMMUNICATION: sensor is transmitting or receiving packet. 
An outline of the protocol implementation is given by Algorithm 1 which describes the execution of a period by a node (denoted by $s_{j}$ for a sensor node indexed by $j$ ). At the beginning a node checks whether it has enough energy (its energy should be greater than a fixed treshold $E_{t h}$ ) to stay active during the next sensing phase. If yes, it exchanges information with all the other nodes belonging to the same subregion: it collects from each node its position coordinates, remaining energy $\left(R E_{j}\right)$, ID, and the number of one-hop neighbors still alive. INFO packet contains two parts: header and payload data. The sensor ID is included in the header, where the header size is 8 bits. The data part includes position coordinates (64 bits), remaining energy (32 bits), and the number of one-hop live neighbors ( 8 bits). Therefore the size of the INFO packet is 112 bits. Once the first phase is completed, the nodes of a subregion choose a leader to take the decision based on the following criteria with decreasing importance: larger number of neighbors, larger remaining energy, and then in case of equality, larger index. After that, if the sensor node is leader, it will solve an integer program (see Section 4). This integer program contains boolean variables $X_{j}$ where $\left(X_{j}=1\right)$ means that sensor $j$ will be active in the next sensing phase. Only sensors with enough remaining energy are involved in the integer program ( $J$ is the set of all sensors involved). As the leader consumes energy (computation energy is denoted by $E^{c o m p}$ ) to solve the optimization problem, it will be included in the integer program only if it has enough energy to achieve the computation and to stay alive during the next sensing phase, that is to say if $R E_{j}>E^{c o m p}+E_{t h}$. Once the optimization problem is solved, each leader will send an ActiveSleep packet to each sensor in the same subregion to indicate it if it has to be active or not. Otherwise, if the sensor is not the leader, it will wait for the ActiveSleep packet to know its state for the coming sensing phase.

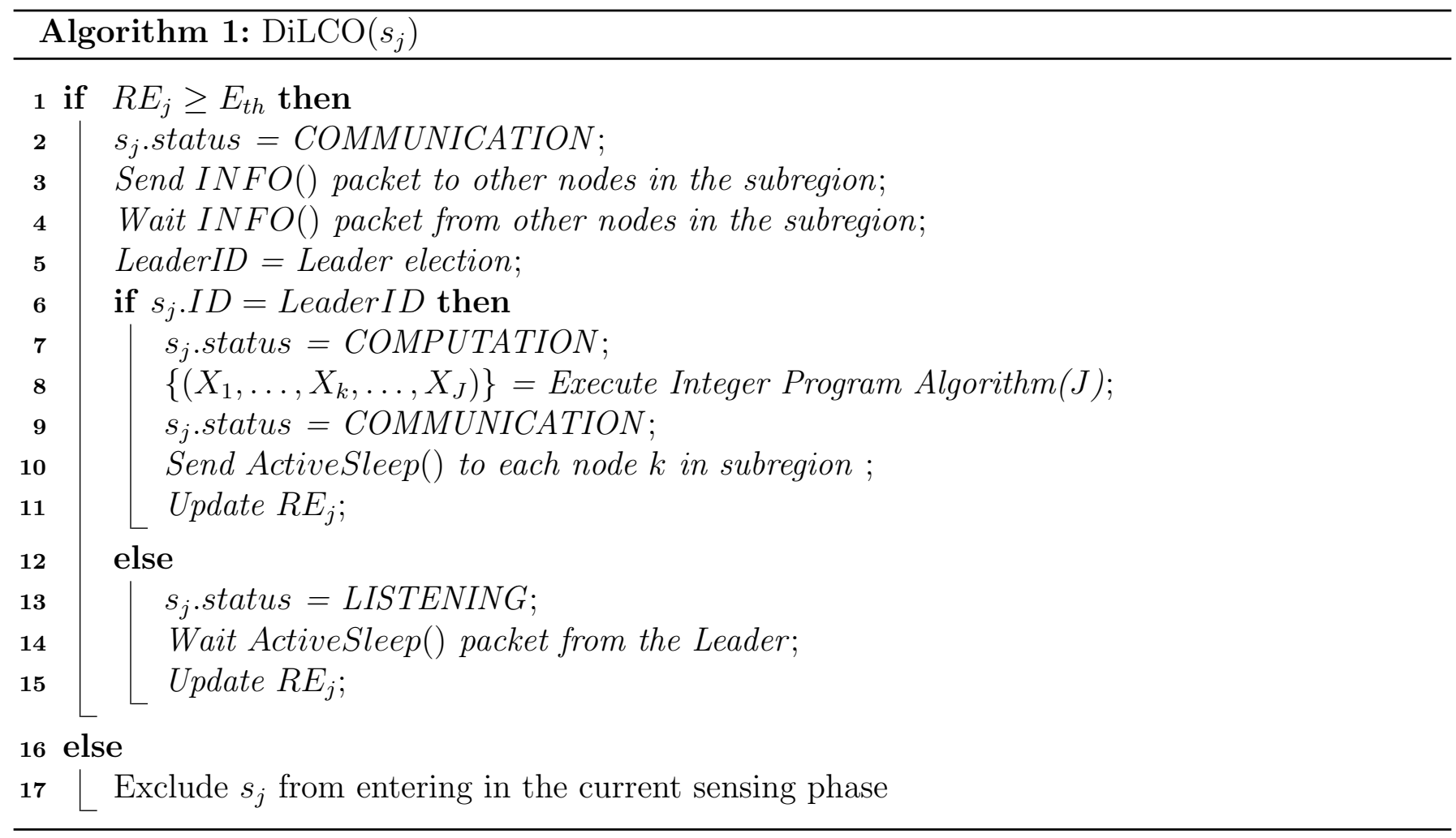

\section{COVERAGE PROBLEM FORMULATION}

We formulate the coverage optimization problem with an integer program. The objective function consists in minimizing the undercoverage and the overcoverage of the area as suggested in [21]. The area coverage problem is expressed as the coverage of a fraction of points called primary points. Details on the choice and the number of primary points can be found in [11]. The set of primary points is denoted by $P$ and the set of alive sensors by $J$. As we consider a boolean disk coverage model, we use the boolean indicator $\alpha_{j p}$ which is equal to 1 if the primary 
point $p$ is in the sensing range of the sensor $j$. The binary variable $X_{j}$ represents the activation or not of the sensor $j$. So we can express the number of active sensors that cover the primary point $p$ by $\sum_{j \in J} \alpha_{j p} * X_{j}$. We deduce the overcoverage denoted by $\Theta_{p}$ of the primary point $p$ :

$$
\Theta_{p}= \begin{cases}0 & \text { if the primary point } \\ \left(\sum_{j \in J} \alpha_{j p} * X_{j}\right)-1 & p \text { is not covered, }\end{cases}
$$

More precisely, $\Theta_{p}$ represents the number of active sensor nodes minus one that cover the primary point $p$. In the same way, we define the undercoverage variable $U_{p}$ of the primary point $p$ as:

$$
U_{p}= \begin{cases}1 & \text { if the primary point } p \text { is not covered, } \\ 0 & \text { otherwise. }\end{cases}
$$

There is, of course, a relationship between the three variables $X_{j}, \Theta_{p}$, and $U_{p}$ which can be formulated as follows :

$$
\sum_{j \in J} \alpha_{j p} X_{j}-\Theta_{p}+U_{p}=1, \forall p \in P
$$

If the point $p$ is not covered, $U_{p}=1, \sum_{j \in J} \alpha_{j p} X_{j}=0$ and $\Theta_{p}=0$ by definition, so the equality is satisfied. On the contrary, if the point $p$ is covered, $U_{p}=0$, and $\Theta_{p}=\left(\sum_{j \in J} \alpha_{j p} X_{j}\right)-1$. Our coverage optimization problem can then be formulated as follows:

$$
\begin{cases}\min \sum_{p \in P}\left(w_{\theta} \Theta_{p}+w_{U} U_{p}\right) & \\ \text { subject to : } & \\ \sum_{j \in J} \alpha_{j p} X_{j}-\Theta_{p}+U_{p}=1, & \forall p \in P \\ \Theta_{p} \in \mathbb{N}, & \forall p \in P \\ U_{p} \in\{0,1\}, & \forall p \in P \\ X_{j} \in\{0,1\}, & \forall j \in J\end{cases}
$$

The objective function is a weighted sum of overcoverage and undercoverage. The goal is to limit the overcoverage in order to activate a minimal number of sensors while simultaneously preventing undercoverage. By choosing $w_{U}$ much larger than $w_{\theta}$, the coverage of a maximum of primary points is ensured. Then for the same number of covered primary points, the solution with a minimal number of active sensors is preferred.

\section{PROTOCOL EVALUATION}

\subsection{Simulation framework}

To assess the performance of our DiLCO protocol, we have used the discrete event simulator OMNeT $++[27]$ to run different series of simulations. Table 1 gives the chosen parameters setting.

Simulations with five different node densities going from 50 to 250 nodes were performed considering each time 25 randomly generated networks, to obtain experimental results which are relevant. The nodes are deployed on a field of interest of $(50 \times 25) \mathrm{m}^{2}$ in such a way that they cover the field with a high coverage ratio.

We chose as energy consumption model the one proposed proposed by [29] and based on [23] with slight modifications. The energy consumed by the communications is added and the part relative to a variable sensing range is removed. We also assume that the nodes have the characteristics of the Medusa II sensor node platform [23]. A sensor node typically consists of four units: a MicroController Unit, an Atmels AVR ATmega103L in case of Medusa II, to perform the computations; a communication (radio) unit able to send and receive messages; a sensing unit to collect data; a power supply which provides the energy consumed by node. Except the battery, all the other unit can be switched off to save energy according to the node 
Table 1: Relevant parameters for network initializing.

\begin{tabular}{c|c}
\hline Parameter & Value \\
\hline Sensing Field & $(50 \times 25) \mathrm{m}^{2}$ \\
Nodes Number & $50,100,150,200$ and 250 nodes \\
Initial Energy & $500-700$ joules \\
Sensing Period & 60 Minutes \\
$E_{t h}$ & 36 Joules \\
$R_{s}$ & $5 \mathrm{~m}$ \\
$w_{\Theta}$ & 1 \\
$w_{U}$ & $|P|^{2}$
\end{tabular}

Table 2: Energy consumption model

\begin{tabular}{|c|c|c|c|c|}
\hline Sensor status & MCU & Radio & Sensing & Power $(\mathrm{mW})$ \\
\hline Listening & ON & ON & ON & 20.05 \\
\hline Active & ON & OFF & ON & 9.72 \\
\hline Sleep & OFF & OFF & OFF & 0.02 \\
\hline Computation & ON & ON & ON & 26.83 \\
\hline
\end{tabular}

status. Table 2 summarizes the energy consumed (in milliWatt per second) by a node for each of its possible status.

Less influent energy consumption sources like when turning on the radio, starting the sensor node, changing the status of a node, etc., will be neglected for the sake of simplicity. Each node saves energy by switching off its radio once it has received its decision status from the corresponding leader (it can be itself). As explained previously in subsection 3.2, two kinds of packets for communication are considered in our protocol: INFO packet and ActiveSleep packet. To compute the energy needed by a node to transmit or receive such packets, we use the equation giving the energy spent to send a 1-bit-content message defined in [23] (we assume symmetric communication costs), and we set their respective size to 112 and 24 bits. The energy required to send or receive a 1-bit-content message is thus equal to $0.2575 \mathrm{~mW}$.

Each node has an initial energy level, in Joules, which is randomly drawn in [500 - 700]. If its energy provision reaches a value below the threshold $E_{t h}=36$ Joules, the minimum energy needed for a node to stay active during one period, it will no longer take part in the coverage task. This value corresponds to the energy needed by the sensing phase, obtained by multiplying the energy consumed in active state $(9.72 \mathrm{~mW})$ by the time in seconds for one period $(3,600$ seconds), and adding the energy for the pre-sensing phases. According to the interval of initial energy, a sensor may be active during at most 20 periods.

In the simulations, we introduce the following performance metrics to evaluate the efficiency of our approach:

- Network Lifetime: we define the network lifetime as the time until the coverage ratio drops below a predefined threshold. We denote by Lifetime ${ }_{95}$ (respectively Lifetime 50 ) the amount of time during which the network can satisfy an area coverage greater than 95\% (respectively 50\%). We assume that the sensor network can fulfill its task until all its nodes have been drained of their energy or it becomes disconnected. Network connectivity is crucial because an active sensor node without connectivity towards a base station cannot transmit any information regarding an observed event in the area that it monitors.

- Coverage Ratio (CR): it measures how well the WSN is able to observe the area of interest. In our case, we discretized the sensor field as a regular grid, which yields the following equation to compute the coverage ratio:

$$
\mathrm{CR}(\%)=\frac{n}{N} \times 100 .
$$

where $n$ is the number of covered grid points by active sensors of every subregions during the current sensing phase and $N$ is the total number of grid points in the sensing field. In our simulations, we have a layout of $N=51 \times 26=1326$ grid points. 
- Energy Consumption: energy consumption (EC) can be seen as the total amount of energy consumed by the sensors during Lifetime 95 or Lifetime 50 , divided by the number of periods. Formally, the computation of EC can be expressed as follows:

$$
\mathrm{EC}=\frac{\sum_{m=1}^{M}\left(E_{m}^{\mathrm{com}}+E_{m}^{\mathrm{list}}+E_{m}^{\mathrm{comp}}+E_{m}^{a}+E_{m}^{s}\right)}{M},
$$

where $M$ corresponds to the number of periods. The total amount of energy consumed by the sensors (EC) comes through taking into consideration four main energy factors. The first one, denoted $E_{m}^{\mathrm{com}}$, represents the energy consumption spent by all the nodes for wireless communications during period $m$. $E_{m}^{\text {list }}$, the next factor, corresponds to the energy consumed by the sensors in LISTENING status before receiving the decision to go active or sleep in period $m . E_{m}^{\text {comp }}$ refers to the energy needed by all the leader nodes to solve the integer program during a period. Finally, $E_{m}^{a}$ and $E_{m}^{s}$ indicate the energy consumed by the whole network in the sensing phase (active and sleeping nodes).

\subsection{Performance analysis}

In this subsection, we first focus on the performance of our DiLCO protocol for different numbers of subregions. We consider partitions of the WSN area into 2, 4, 8, 16, and 32 subregions. Thus the DiLCO protocol is declined in five versions: DiLCO-2, DiLCO-4, DiLCO-8, DiLCO-16, and DiLCO-32. Simulations without partitioning the area of interest, cases which correspond to a centralized approach, are not presented because they require high execution times to solve the integer program and therefore consume too much energy.

We compare our protocol to two other approaches. The first one, called DESK and proposed by [29] is a fully distributed coverage algorithm. The second one, called GAF [31], consists in dividing the region into fixed squares. During the decision phase, in each square, one sensor is chosen to remain active during the sensing phase.

\subsubsection{Coverage ratio}

Figure 2 shows the average coverage ratio for 150 deployed nodes. It can be seen that both DESK and GAF provide a coverage ratio which is slightly better compared to DiLCO in the first thirty periods. This can be easily explained by the number of active nodes: the optimization process of our protocol activates less nodes than DESK or GAF, resulting in a slight decrease of the coverage ratio. In case of DiLCO-2 (respectively DiLCO-4), the coverage ratio exhibits a fast decrease with the number of periods and reaches zero value in period 18 (respectively 46), whereas the other versions of DiLCO, DESK, and GAF ensure a coverage ratio above $50 \%$ for subsequent periods. We believe that the results obtained with these two methods can be explained by a high consumption of energy and we will check this assumption in the next subsection.

Concerning DiLCO-8, DiLCO-16, and DiLCO-32, these methods seem to be more efficient than DESK and GAF, since they can provide the same level of coverage (except in the first periods where DESK and GAF slightly outperform them) for a greater number of periods. In fact, when our protocol is applied with a large number of subregions (from 8 to 32 regions), it activates a restricted number of nodes, and thus enables the extension of the network lifetime.

\subsubsection{Energy consumption}

Based on the results shown in Figure 2, we focus on the DiLCO-16 and DiLCO-32 versions of our protocol, and we compare their energy consumption with the DESK and GAF approaches. For each sensor node we measure the energy consumed according to its successive status, for different network densities. We denote by Protocol/50 (respectively Protocol/95) the amount of energy consumed while the area coverage is greater than $50 \%$ (repectively $95 \%$ ), where Protocol is one of the four protocols we compare. Figure 3 presents the energy consumptions observed 


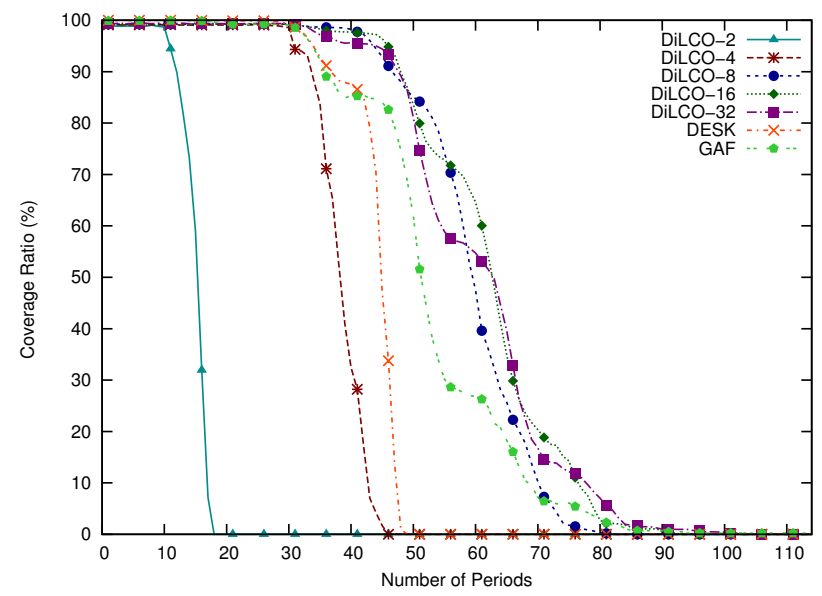

Figure 2: Coverage ratio

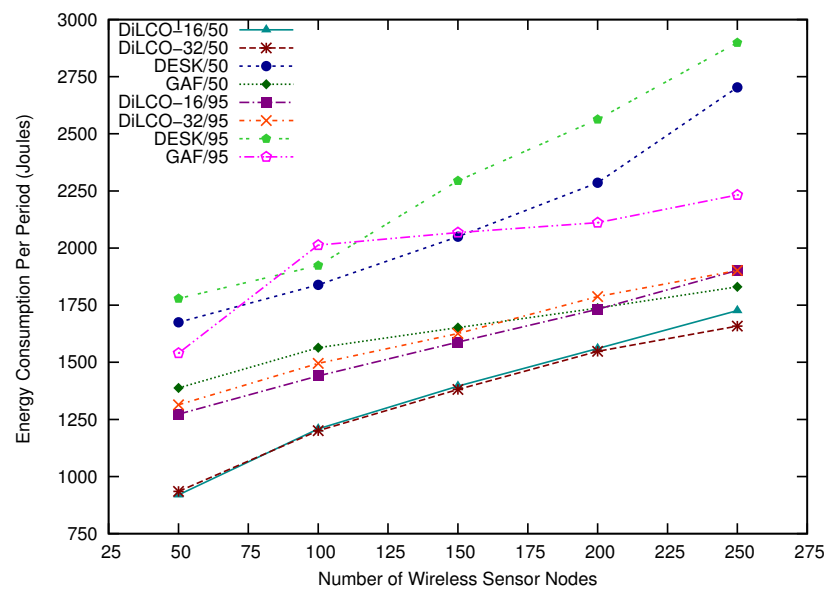

Figure 3: Energy consumption per period

for network sizes going from 50 to 250 nodes. Let us notice that the same network sizes will be used for the different performance metrics.

The results depict the good performance of the different versions of our protocol. Indeed, the protocols DiLCO-16/50, DiLCO-32/50, DiLCO-16/95, and DiLCO-32/95 consume less energy than their DESK and GAF counterparts for a similar level of area coverage. This observation reflects the larger number of nodes set active by DESK and GAF.

Now, if we consider a same protocol, we can notice that the average consumption per period increases slightly for our protocol when increasing the level of coverage and the number of node, whereas it increases more largely for DESK and GAF. In case of DiLCO, it means that even if a larger network allows to improve the number of periods with a minimum coverage level value, this improvement has a higher energy cost per period due to communication overhead and a more difficult optimization problem. However, in comparison with DESK and GAF, our approach has a reasonable energy overcost.

\subsubsection{Execution time}

Another interesting point to investigate is the evolution of the execution time with the size of the WSN and the number of subregions. Therefore, we report for every version of our protocol the average execution times in seconds needed to solve the optimization problem for different WSN sizes. The execution times are obtained on a laptop DELL which has an Intel Core i3 $2370 \mathrm{M}(2.4 \mathrm{GHz})$ dual core processor and a MIPS rating equal to 35330 . The corresponding execution times on a MEDUSA II sensor node are then extrapolated according to the MIPS rate of the Atmels AVR ATmega103L microcontroller $(6 \mathrm{MHz})$, which is equal to 6 , by multiplying the laptop times by $\left(\frac{35330}{2} \times \frac{1}{6}\right)$. The expected times on a sensor node are reported on Figure 4.

Figure 4 shows that DiLCO-32 has very low execution times in comparison with other DiLCO 


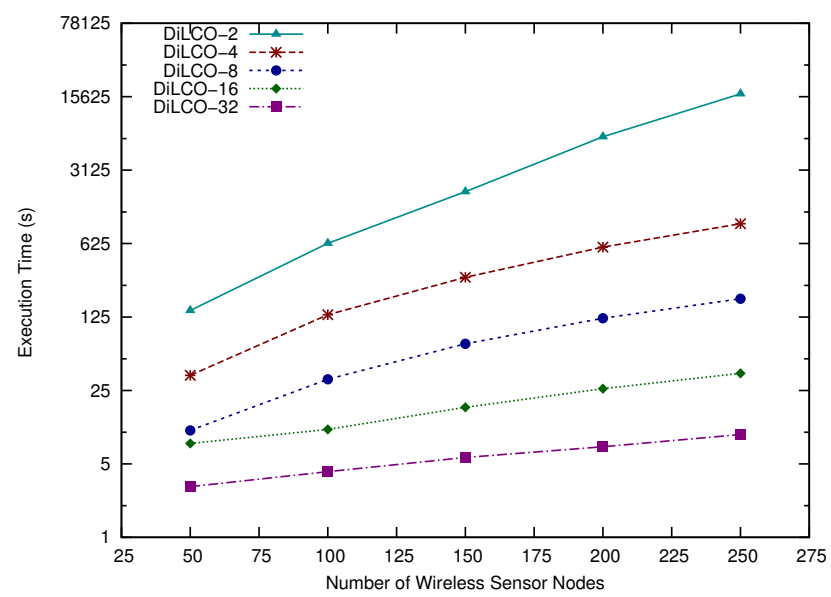

Figure 4: Execution time in seconds

versions, because the activity scheduling is tackled by a larger number of leaders and each leader solves an integer problem with a limited number of variables and constraints. Conversely, DiLCO-2 requires to solve an optimization problem with half of the network nodes and thus presents a high execution time. Nevertheless if we refer to Figure 2, we observe that DiLCO-32 is slightly less efficient than DilCO-16 to maintain as long as possible high coverage. In fact an excessive subdivision of the area of interest prevents it to ensure a good coverage especially on the borders of the subregions. Thus, the optimal number of subregions can be seen as a trade-off between execution time and coverage performance.

\subsubsection{Network lifetime}

In the next figure, the network lifetime is illustrated. Obviously, the lifetime increases with the network size, whatever the considered protocol, since the correlated node density also increases. A high network density means a high node redundancy which allows to turn-off many nodes and thus to prolong the network lifetime.

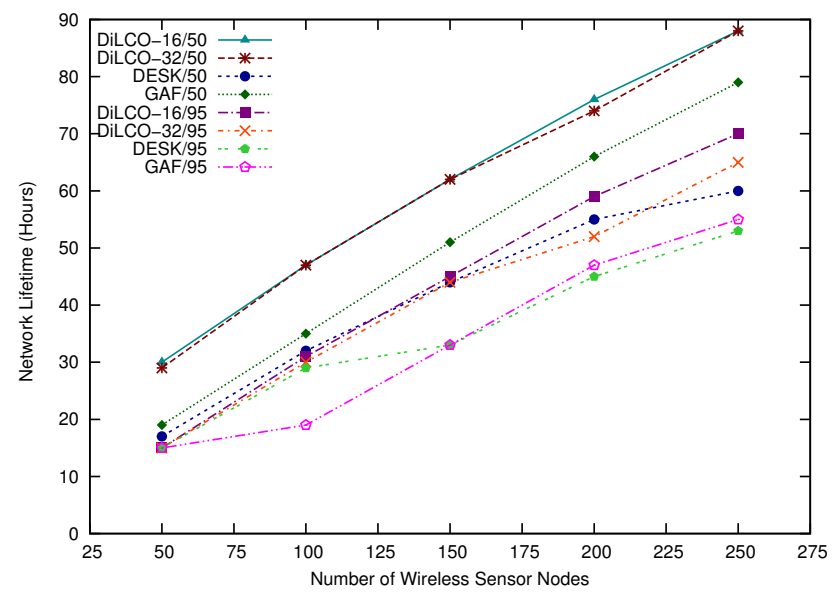

Figure 5: Network lifetime

As highlighted by Figure 5, when the coverage level is relaxed (50\%) the network lifetime also improves. This observation reflects the fact that the higher the coverage performance, the more nodes must be active to ensure the wider monitoring. For a similar level of coverage, DiLCO outperforms DESK and GAF for the lifetime of the network. More specifically, if we focus on the larger level of coverage (95\%) in the case of our protocol, the subdivision in 16 subregions seems to be the most appropriate. 


\section{CONCLUSION AND FUTURE WORK}

A crucial problem in WSN is to schedule the sensing activities of the different nodes in order to ensure both coverage of the area of interest and longer network lifetime. The inherent limitations of sensor nodes, in energy provision, communication and computing capacities, require protocols that optimize the use of the available resources to fulfill the sensing task. To address this problem, this paper proposes a two-step approach. Firstly, the field of sensing is divided into smaller subregions using the concept of divide-and-conquer method. Secondly, a distributed protocol called Distributed Lifetime Coverage Optimization is applied in each subregion to optimize the coverage and lifetime performances. In a subregion, our protocol consists in electing a leader node which will then perform a sensor activity scheduling. The challenges include how to select the most efficient leader in each subregion and the best representative set of active nodes to ensure a high level of coverage. To assess the performance of our approach, we compared it with two other approaches using many performance metrics like coverage ratio or network lifetime. We have also studied the impact of the number of subregions chosen to subdivide the area of interest, considering different network sizes. The experiments show that increasing the number of subregions improves the lifetime. The more subregions there are, the more robust the network is against random disconnection resulting from dead nodes. However, for a given sensing field and network size there is an optimal number of subregions. Therefore, in case of our simulation context a subdivision in 16 subregions seems to be the most relevant. The optimal number of subregions will be investigated in the future.

\section{ACKNOWLEDGEMENTS}

As a Ph.D. student, Ali Kadhum IDREES would like to gratefully acknowledge the University of Babylon - IRAQ for the financial support and Campus France for the received support. This paper is also partially funded by the Labex ACTION program (contract ANR-11-LABX-01-01).

\section{References}

[1] Ian F Akyildiz, Weilian Su, Yogesh Sankarasubramaniam, and Erdal Cayirci. A survey on sensor networks. IEEE Communications magazine, 40(8):102-114, 2002.

[2] Ian F Akyildiz and Mehmet Can Vuran. Wireless sensor networks, volume 4. John Wiley \& Sons, 2010.

[3] P. Berman and G. Calinescu. Power efficient monitoring management in sensor networks. In Proceedings of IEEE Wireless Communication and Networking Conference (WCNC'04), pages 2329-2334, 2004.

[4] Mihaela Cardei and Ding-Zhu Du. Improving wireless sensor network lifetime through power aware organization. Wireless Networks, 11(3):333-340, 2005.

[5] Mihaela Cardei, My T Thai, Yingshu Li, and Weili Wu. Energy-efficient target coverage in wireless sensor networks. In INFOCOM 2005. 24th Annual Joint Conference of the IEEE Computer and Communications Societies. Proceedings IEEE, volume 3, pages 1976-1984. IEEE, 2005.

[6] Fabian Castaño, André Rossi, Marc Sevaux, and Nubia Velasco. A column generation approach to extend lifetime in wireless sensor networks with coverage and connectivity constraints. Computers \& Operations Research, 2013.

[7] Marco Conti and Silvia Giordano. Mobile ad hoc networking: milestones, challenges, and new research directions. Communications Magazine, IEEE, 52(1):85-96, 2014.

[8] Xiu Deng, Dongxiao Yu Jiguo Yu, and Congcong Chen. Transforming area coverage to target coverage to maintain coverage and connectivity for wireless sensor networks. International Journal of Distributed Sensor Networks, 2012, 2012. 
[9] Karine Deschinkel. A column generation based heuristic to extend lifetime in wireless sensor network. Sensors \& Transducers Journal, 14-2:242-253, 2012.

[10] Wendi Rabiner Heinzelman, Anantha Chandrakasan, and Hari Balakrishnan. Energyefficient communication protocol for wireless microsensor networks. In 33rd Annual Hawaii International Conference on System Sciences (HICSS-33), 4-7 January, 2000, Maui, Hawaii, USA. IEEE Computer Society, 2000.

[11] Ali Kadhum Idrees, Karine Deschinkel, Michel Salomon, and Raphaël Couturier. Coverage and lifetime optimization in heterogeneous energy wireless sensor networks. In ICN 2014, The Thirteenth International Conference on Networks, pages 49-54, 2014.

[12] N. Jaggi and A.A. Abouzeid. Energy-efficient connected covereage in wireless sensor networks. In Proceeding of 4th Asian International Mobile Computing Conference AMOC2006, 2006 .

[13] Hyunbum Kim and Jorge A Cobb. Maximum lifetime of reinforced barrier-coverage in wireless sensor networks. In Networks (ICON), 2013 19th IEEE International Conference on, pages 1-6. IEEE, 2013.

[14] Santosh Kumar, Ten H. Lai, and Anish Arora. Barrier coverage with wireless sensors. In Proceedings of the 11th Annual International Conference on Mobile Computing and Networking, MobiCom '05, pages 284-298, New York, NY, USA, 2005. ACM.

[15] Mo Li and Athanasios V Vasilakos. A survey on topology control in wireless sensor networks: Taxonomy, comparative study, and open issues. Proceedings of the IEEE, 101(12), 2013.

[16] Hui Ling and Taieb Znati. Energy efficient adaptive sensing for dynamic coverage in wireless sensor networks. In Wireless Communications and Networking Conference, 2009. WCNC 2009. IEEE, pages 1-6. IEEE, 2009.

[17] Manju and Arun K Pujari. High-energy-first (hef) heuristic for energy-efficient target coverage problem. International Journal of Ad Hoc, Sensor $\&$ Ubiquitous Computing, 2(1), 2011.

[18] S. Misra, M. P. Kumar, and M. S. Obaidat. Connectivity preserving localized coverage algorithm for area monitoring using wireless sensor networks. Computer Communications, 34(12):1484-1496, 2011.

[19] A. Nayak and I. Stojmenovic. Wireless Sensor and Actuator Networks: Algorithms and Protocols for Scalable Coordination and Data Communication. John Wiley and Sons, Inc, 2010.

[20] T.V. Padmavathy and M. Chitra. Extending the network lifetime of wireless sensor networks using residual energy extraction-hybrid scheduling algorithm. Int. J. of Communications, Network and System Sciences, 3(1):98-106, 2010.

[21] F. Pedraza, A. L. Medaglia, and A. Garcia. Efficient coverage algorithms for wireless sensor networks. In Proceedings of the 2006 Systems and Information Engineering Design Symposium, pages 78-83, 2006.

[22] Yipeng $\mathrm{Qu}$ and Stavros V Georgakopoulos. A distributed area coverage algorithm for maintenance of randomly distributed sensors with adjustable sensing range. In Global Communications Conference (GLOBECOM), 2013 IEEE, pages 286-291. IEEE, 2013.

[23] Vijay Raghunathan, Curt Schurgers, Sung Park, and Mani B Srivastava. Energy-aware wireless microsensor networks. Signal Processing Magazine, IEEE, 19(2):40-50, 2002.

[24] K. Ramesh and K. Somasundaram. A comparative study of clusterhead selection algorithms in wireless sensor networks. International Journal of Computer Science and Engineering Survey, 2(4), November 2011.

[25] Tifenn Rault, Abdelmadjid Bouabdallah, and Yacine Challal. Energy efficiency in wireless sensor networks: A top-down survey. Computer Networks, 67:104-122, 2014. 
[26] André Rossi, Alok Singh, and Marc Sevaux. An exact approach for maximizing the lifetime of sensor networks with adjustable sensing ranges. Computers $\&$ Operations Research, 39(12):3166-3176, 2012.

[27] A. Varga. Omnet++ discrete event simulation system. Available: http://www.omnetpp.org, 2003.

[28] C. T. Vu. Distributed Energy-Efficient solutions for area coverage problems in Wireless Sensor Networks. PhD thesis, Georgia State University, 2009.

[29] Chinh Vu, Shan Gao, Wiwek Deshmukh, and Yingshu Li. Distributed energy-efficient scheduling approach for k-coverage in wireless sensor networks. MILCOM, 0:1-7, 2006.

[30] Xiaofei Xing, Jie Li, and Guojun Wang. Integer programming scheme for target coverage in heterogeneous wireless sensor networks. In Mobile Ad-hoc and Sensor Networks (MSN), 2010 Sixth International Conference on, pages 79-84, Dec 2010.

[31] Ya Xu, John Heidemann, and Deborah Estrin. Geography-informed energy conservation for ad hoc routing. In Proceedings of the 7th annual international conference on Mobile computing and networking, pages 70-84. ACM, 2001.

[32] Changlin Yang and Kwan-Wu Chin. Novel algorithms for complete targets coverage in energy harvesting wireless sensor networks. Communications Letters, IEEE, 18(1):118121, January 2014.

[33] Changlin Yang and Kwan-Wu Chin. A novel distributed algorithm for complete targets coverage in energy harvesting wireless sensor networks. In IEEE ICC 2014-Ad-hoc and Sensor Networking Symposium, pages 361-366. IEEE, 2014.

[34] Mengmeng Yang and Jie Liu. A maximum lifetime coverage algorithm based on linear programming. Journal of Information Hiding an dMultimedia Signal Processing, Ubiquitous International, 5(2):296-301, 2014.

[35] H. Zhang and J. C. Hou. Maintaining sensing coverage and connectivity in large sensor networks. Ad Hoc \& Sensor Wireless Networks, 1(1-2), 2005.

[36] Dimitrios Zorbas, Dimitris Glynos, Panayiotis Kotzanikolaou, and Christos Douligeris. Solving coverage problems in wireless sensor networks using cover sets. Ad Hoc Networks, 8(4):400-415, 2010. 